\title{
ON AN ARCWISE CONNECTED SUBGROUP OF A LIE GROUP
}

MORIKUNI GOTO

1. Introduction. Let $G$ be a Lie group, and let (5) be the Lie algebra of $G$. A continuous isomorphic image of a connected Lie group in $G$ is said to be an analytic subgroup of $G$. There is a one-toone correspondence between the set of all subalgebras of $(5)$ and the set of all analytic subgroups of $G$. An analytic subgroup is naturally arcwise connected. The main purpose of this paper is to give a detailed proof of the following known theorem.

TheOREM. Let $G$ be a Lie group, and let $A$ be an arcwise connected subgroup of $G$. Then $A$ is an analytic subgroup.

This theorem was first proved by M. Kuranishi and the late H. Yamabe [Osaka Math. J. 2 (1950), 13-14] in different ways. However, the proof of Kuranishi has never been published, and that of Yamabe seems too short to be completely understandable. The proof presented here follows more or less familiar lines.

In the end of the paper, some of the direct corollaries of the theorem shall be given for the convenience of the reader.

2. Outline of the proof. Let $G$ be a Lie group, and let (s) be the associated Lie algebra. $e$ denotes the unit element of $G$.

Definition. Let $A$ be an arcwise connected subgroup of $G$. Then an element $L$ of $(S)$ is said to be accessible (by $A$ ) if for any neighborhood $U$ of $e$, there exists an arc (=continuous curve) $\alpha(t), 0 \leqq t \leqq 1$, in $A$ such that $\alpha(0)=e$ and $\alpha(t) \in \exp (t L) \cdot U$ for $0 \leqq t \leqq 1$.

We denote by $\mathfrak{S}$ the set of all accessible $L$ 's. We shall now divide the proof of the theorem into three steps:

(A) $\mathfrak{S}$ is a subalgebra of $(5)$.

(B) Denoting by $H$ the analytic subgroup of $G$ corresponding to $\mathfrak{S}$, we have $H \supset A$.

(C) $H=A$.

3. Proof of $(\mathbf{A})$.

(i) Let $L$ be an element of $\mathfrak{S}$.

Let $s$ be a real number between 0 and 1 . It is clear that $s L \in \mathfrak{S}$.

Let $U$ be a neighborhood of $e$. Then we can find a neighborhood $V$ of $e$ such that $V=V^{-1}$ and $\exp (t L) \cdot V \cdot \exp (-t L) \subset U$ for $0 \leqq t \leqq 1$.

Received by the editors September 11, 1967. 
Let $\alpha(t)$ be an arc in $A$ with $\alpha(0)=e$ and $\alpha(t) \in \exp (t L) \cdot V$. Then $\alpha(t)^{-1} \in V^{-1} \cdot \exp (-t L) \subset \exp (-t L) \cdot U$. Hence $-L \in \mathfrak{S}$.

Let $k$ be a positive integer. For a given neighborhood $U$ of $e$, we can find a neighborhood $V$ of $e$ such that

$$
\begin{aligned}
a_{1} \in \exp \left(t_{1} L\right) \cdot V, a_{2} \in & \exp \left(t_{2} L\right) \cdot V, \cdots, a_{k} \in \exp \left(t_{k} L\right) \cdot V, \\
& 0 \leqq t_{1} \leqq 1,0 \leqq t_{2} \leqq 1, \cdots, 0 \leqq t_{k} \leqq 1,
\end{aligned}
$$

implies that $a_{1} a_{2} \cdots a_{k} \in \exp \left(\left(t_{1}+t_{2}+\cdots+t_{k}\right) L\right) \cdot U$. We take an $\operatorname{arc} \beta(t)$ in $A$ with $\beta(0)=e$ and $\beta(t) \in \exp (t L) \cdot V$ and set

$$
\gamma(t)=\beta(1)^{[k t]} \beta(k t-[k t]), \quad 0 \leqq t \leqq 1,
$$

where [ ] denotes the Gaussian symbol. Then $\gamma(t)$ is an arc in $A$, $\gamma(0)=e$, and

$$
\gamma(t) \in(\exp (L))^{[k t]} \cdot \exp ((k t-[k t]) L) \cdot U=\exp (t k L) \cdot U .
$$

Hence $k L \in \mathfrak{S}$.

Thus we have proved that $L \in \mathfrak{S}$ implies that $\boldsymbol{R} L \subset \mathfrak{S}$, where $\boldsymbol{R}$ denotes the field of real numbers.

(ii) Next we shall prove that $X \in \mathfrak{S}$ and $Y \in \mathfrak{S}$ implies that $X+Y \in \mathfrak{S}$ and $[X, Y] \in \mathfrak{S}$. In view of the preceding result, it is sufficient to prove this under the additional assumption that $X$ and $Y$ are arbitrary close to 0 . For such $X$ and $Y$ we have

$$
\exp (t(X+Y))=\lim _{m \rightarrow \infty}\left(\exp \frac{t X}{m} \cdot \exp \frac{t Y}{m}\right)^{m}, \quad 0 \leqq t \leqq 1,
$$

where the convergence is uniform on $t$.

For a given neighborhood $U$ of $e$, we take a neighborhood $V$ of $e$ with $V^{2} \subset U$. Then we can find an integer $k$ such that

$$
\left(\exp \frac{t X}{k} \cdot \exp \frac{t Y}{k}\right)^{k} \in \exp (t(X+Y)) \cdot V, \quad 0 \leqq t \leqq 1 .
$$

Next let us take a neighborhood $W$ of $e$ so small that $a \in \exp (t X / k) \cdot W$ and $b \in \exp (t Y / k) \cdot W(0 \leqq t \leqq 1)$ implies that

$$
(a b)^{k} \in(\exp (t X / k) \cdot \exp (t Y / k))^{k} \cdot V .
$$

For this $W$ we can find $\operatorname{arcs} \alpha(t)$ and $\beta(t)$ in $A$ with

$$
\begin{array}{lll}
\alpha(0)=e, & \alpha(t) \in \exp (t X / k) \cdot W, & 0 \leqq t \leqq 1, \\
\beta(0)=e, & \beta(t) \in \exp (t Y / k) \cdot W, & 0 \leqq t \leqq 1 .
\end{array}
$$

Set $\gamma(t)=(\alpha(t) \beta(t))^{k}, 0 \leqq t \leqq 1$. Then $\gamma(t)$ is also an arc in $A$ with $\gamma(0)=e$ and 
$\gamma(t) \in\left(\exp \frac{t X}{k} \cdot \exp \frac{t Y}{k}\right)^{k} \cdot V \subset \exp (t(X+Y)) \cdot V^{2} \subset \exp (t(X+Y)) \cdot U$

Hence $X+Y$ is accessible.

Similarly using the uniform convergence of

$$
\begin{array}{r}
\lim _{m \rightarrow \infty}\left(\exp \frac{\sqrt{ } t X}{m} \cdot \exp \frac{\sqrt{ } t Y}{m} \cdot \exp \frac{-\sqrt{ } t X}{m} \cdot \exp \frac{-\sqrt{ } t Y}{m}\right)^{m^{2}}=\exp (t[X, Y]), \\
0 \leqq t \leqq 1,
\end{array}
$$

we can prove that $X, Y \in \mathfrak{S}$ implies that $[X, Y] \in \mathfrak{S}$.

4. Proof of (B). We fix a basis $L_{1}, L_{2}, \cdots, L_{n}$ of (S), and use the euclidean norm with respect to the basis. That is, we define

$\|X\|=\left(x_{1}^{2}+x_{2}^{2}+\cdots+x_{n}^{2}\right)^{1 / 2}$ for $X=x_{1} L_{1}+x_{2} L_{2}+\cdots+x_{n} L_{n}$.

We set

$$
V_{m}=\{\exp X ; X \in \mathbb{B} \text { and } m\|X\|<1\} \quad \text { for } m=1,2, \cdots
$$

$\left\{V_{m}\right\}$ forms a complete system of neighborhoods of $e$. Next we take a linear subspace $\mathfrak{P}$ of $\mathfrak{B}$ which is complementary to $\mathfrak{S}: \mathfrak{S}=\mathfrak{S}+\mathfrak{B}$ and $\mathfrak{S} \cap \mathfrak{B}=\{0\}$.

For each $m$ we can choose a neighborhood $W_{m}$ of $e$ in $V_{m}$ such that every element $a$ of $W_{m}$ can be written uniquely in the form

$$
a=\exp X \cdot \exp Y
$$

where $X \in \mathfrak{S}, m\|X\|<1$ and $Y \in \mathfrak{P}, m\|Y\|<1$. We may assume moreover $W_{m} \supset W_{m+1}$ for $m=1,2, \cdots$. Let us denote by $A_{m}$ the arcwise connected component of $W_{m} \cap A$ containing $e$.

Lemma 1. Let $A$ be an arcwise connected topological group, and let $U$ be a neighborhood of the unit element in $A$. Then the arcwise connected component $U^{a}$ of $U$ containing the unit element is a generating system.

By the above obvious lemma, in order to prove (B) it suffices to show that some $A_{m}$ is contained in $H$.

Suppose there is no such $m$. Then for each $m$ we can find an element $a_{m}$ in $A_{m}$ with

$$
a_{m}=\exp X_{m} \cdot \exp Y_{m}, \quad X_{m} \in \mathfrak{S}, \quad 0 \neq Y_{m} \in \mathfrak{P} .
$$

Since $\left\{Y_{m} /\left\|Y_{m}\right\| ; m=1,2, \cdots\right\}$ is a sequence of vectors of norm one, we can find a convergent subsequence. Since $\left\{A_{m} ; m=1,2, \cdots\right\}$ 
is a monotone decreasing sequence of sets, we may assume, without changing the notations, that $\lim _{m \rightarrow \infty} Y_{m} /\left\|Y_{m}\right\|=Y \in \mathfrak{B}$. Setting $p_{m}=\left[1 /\left\|Y_{m}\right\|\right]$, we have $\lim _{m \rightarrow \infty} p_{m} Y_{m}=Y$.

Let $Z$ be a neighborhood of $e$. We can find a neighborhood $Z^{\prime}$ of $e$ with $Z^{\prime} a_{m} \subset a_{m} Z$ and $Z^{\prime} \subset V_{m}$. Since $X_{m} \in \mathfrak{S}$, we can find an arc $\gamma$ in $A$ such that $\gamma(0)=e$ and $\gamma(t) \in \exp \left(-t X_{m}\right) \cdot Z^{\prime}$ for $0 \leqq t \leqq 1$. Next let us take an arc $\delta$ in $A_{m}$ with $\delta(0)=e$ and $\delta(1)=a_{m}$. We set $\beta(t)$ $=\gamma(t) \delta(t)$. Then $\beta$ is an arc in $A, \beta(0)=e, \beta(1)=\gamma(1) \delta(1) \in \exp \left(-X_{m}\right)$ - $Z^{\prime} a_{m} \subset \exp Y_{m} \cdot Z$, and $\beta(t) \in V_{m} Z^{\prime} A_{m} \subset V_{m}^{3}$. Since $Z$ is arbitrary and $m\left\|Y_{m}\right\|<1$, we can take $Z$ so small that $\beta(1)=\exp Y_{m}^{\prime}, p_{m}^{2}\left\|Y_{m}-Y_{m}^{\prime}\right\|<1$ and $m\left\|Y_{m}^{\prime}\right\|<1$.

Thus for every $m$ there corresponds an $\operatorname{arc} \beta_{m}$ in $A \cap V_{m}^{3}$ such that $\beta_{m}(0)=e, \beta_{m}(1)=\exp Y_{m}^{\prime}$, and $\lim _{m \rightarrow \infty} p_{m} Y_{m}^{\prime}=Y$. Now we define an arc $\alpha_{m}$ in $A$ by

$$
\alpha_{m}(t)=\beta_{m}(1)^{\left[t p_{m}\right]} \beta_{m}\left(t p_{m}-\left[t p_{m}\right]\right), \quad 0 \leqq t \leqq 1,
$$

then

$$
\begin{aligned}
\alpha_{m}(t) & =\exp \left(\left[t p_{m}\right] Y_{m}^{\prime}\right) \cdot \beta_{m}\left(t p_{m}-\left[t p_{m}\right]\right) \\
& =\exp \left(t p_{m} Y_{m}^{\prime}\right) \cdot \exp \left(\left(\left[t p_{m}\right]-t p_{m}\right) Y_{m}^{\prime}\right) \cdot \beta_{m}\left(t p_{m}-\left[t p_{m}\right]\right) \\
& \in \exp \left(t p_{m} Y_{m}^{\prime}\right) \cdot V_{m} \cdot V_{m}^{3}=\exp \left(t p_{m} Y_{m}^{\prime}\right) \cdot V_{m}^{4} .
\end{aligned}
$$

Now let us prove that $Y \in \mathfrak{S}$. Let $U$ be a given neighborhood of $e$. We can find a positive integer $k$ with $V_{\mathbf{k}}^{5} \subset U$. Since $\exp \left(t p_{m} Y_{m}^{\prime}\right)$ converges uniformly to $\exp (t Y)$ for $0 \leqq t \leqq 1$, we can find $h>k$ such that $\exp \left(t p_{h} Y_{h}^{\prime}\right) \in \exp (t Y) \cdot V_{k}$. Hence

$$
\alpha_{h}(t) \in \exp \left(t p_{h} Y_{h}^{\prime}\right) \cdot V_{h}^{4} \subset \exp (t Y) \cdot V_{k} \cdot V_{h}^{4} \subset \exp (t Y) \cdot U .
$$

This is a contradiction because $Y \in \mathfrak{P}$ and $\|Y\|=1$.

\section{Proof of (C).}

Lemma 2. Let I denote the closed interval $[-1,1]$. Let $r$ be a positive integer. Let $f$ be a continuous map from $I^{r}$ into $R^{r}$ such that $\|f(x)-x\|$ $\leqq \frac{1}{2}$ for all $x$ in $I^{r}$, where \|\| denotes the euclidean norm in $R^{r}$. Then $f\left(I^{r}\right)$ contains the origin 0 as an interior point.

Proof. Take $a$ in $R^{r}$ with $2\|a\| \leqq 1$ and set $g(x)=x-f(x)+a$ for $x \in I^{r}$. Since $\|g(x)\| \leqq\|x-f(x)\|+\|a\| \leqq 1, g$ is a continuous map from $I^{r}$ into itself. Hence by the Brouwer fixed point theorem there exists an $x_{0}$ in $I^{r}$ with $g\left(x_{0}\right)=x_{0}$, i.e. $f\left(x_{0}\right)=a$. Thus we have shown that $f\left(I^{r}\right)$ contains the disc of radius $\frac{1}{2}$ about 0 . Q.E.D.

Let us choose a basis $X_{1}, X_{2}, \cdots, X_{r}$ of $H$ such that in 


$$
\begin{aligned}
& V=\left\{\exp \left(t_{1} X_{1}\right) \cdot \exp \left(t_{2} X_{2}\right)\right. \cdots \exp \left(t_{r} X_{r}\right) \\
&\left.-2<t_{1}<2,-2<t_{2}<2, \cdots,-2<t_{r}<2\right\}, \\
& \exp \left(t_{1} X_{1}\right) \cdot \exp \left(t_{2} X_{2}\right) \cdots \cdot \exp \left(t_{r} X_{r}\right) \mapsto\left(t_{1}, t_{2}, \cdots, t_{r}\right)
\end{aligned}
$$

gives a holomorphic coordinate system at $e$ of the Lie group $H$. Let us take a neighborhood $W$ of $e$ so small that $\exp \left(t_{1} X_{1}\right) \cdot \exp \left(t_{2} X_{2}\right) \cdot \ldots$ $\cdot \exp \left(t_{r} X_{r}\right) \cdot W \subset V$ for $\quad-1 \leqq t_{1} \leqq 1, \quad-1 \leqq t_{2} \leqq 1, \cdots, \quad-1 \leqq t_{r} \leqq 1$, and if

$$
\begin{aligned}
\exp \left(s_{1} X_{1}\right) \cdot \exp \left(s_{2} X_{2}\right) \cdots & \exp \left(s_{r} X_{r}\right) \\
& \quad \in \exp \left(t_{1} X_{1}\right) \cdot \exp \left(t_{2} X_{2}\right) \cdots \cdot \exp \left(t_{r} X_{r}\right) \cdot W
\end{aligned}
$$

for $-1 \leqq t_{1} \leqq 1,-1 \leqq t_{2} \leqq 1, \cdots,-1 \leqq t_{r} \leqq 1$ then $\left(s_{1}-t_{1}\right)^{2}+\left(s_{2}-t_{2}\right)^{2}$ $+\cdots+\left(s_{r}-t_{r}\right)^{2} \leqq 1 / 4$. Next, we take a neighborhood $Z$ of $e$ such that

$\exp \left(t_{1} X_{1}\right) \cdot Z \cdot \exp \left(t_{2} X_{2}\right) \cdot Z \cdot \cdots \cdot \exp \left(t_{r} X_{r}\right) \cdot Z$

$$
C \exp \left(t_{1} X_{1}\right) \cdot \exp \left(t_{2} X_{2}\right) \cdots \cdot \exp \left(t_{r} X_{r}\right) \cdot W .
$$

For this $Z$ we can find $\operatorname{arcs} \alpha_{1}(t), \alpha_{2}(t), \cdots, \alpha_{r}(t)$ in $A$ such that $\alpha_{i}(0)=e$ and $\alpha_{i}(t) \in \exp \left(t X_{i}\right) \cdot Z$ for $0 \leqq t \leqq 1$, and $i=1,2, \cdots, r$.

Then $\alpha_{1}\left(t_{1}\right) \alpha_{2}\left(t_{2}\right) \cdots \alpha_{r}\left(t_{r}\right),\left(t_{1}, t_{2}, \cdots, t_{r}\right) \in I^{r}$, is in $V$, and is written in the form

$$
\exp \left(f_{1} X_{1}\right) \cdot \exp \left(f_{2} X_{2}\right) \cdots \cdot \exp \left(f_{r} X_{r}\right)
$$

where $f_{i}=f_{i}\left(t_{1}, t_{2}, \cdots, t_{r}\right)$ is a continuous function defined in $I^{r}$. Then $f(t)=\left(f_{1}(t), f_{2}(t), \cdots, f_{r}(t)\right)$ for $t=\left(t_{1}, t_{2}, \cdots, t_{r}\right)$ defines a continuous map from $I^{r}$ into $R^{r}$, with $\|t-f(t)\| \leqq 1 / 2$. Therefore by Lemma 2 $\left\{\alpha_{1}\left(t_{1}\right) \alpha_{2}\left(t_{2}\right) \cdots \alpha_{r}\left(t_{r}\right) ;\left(t_{1}, t_{2}, \cdots, t_{r}\right) \in I^{r}\right\}$ contains a neighborhood of $e$ in $H$. Hence $H=A$.

\section{Corollaries.}

Corollary 1. Let $G$ be a Lie group. Let $H$ be an analytic subgroup and $K$ a subgroup of $G$. If $H K=K H$, then the commutator subgroup $[H, K]$ is an analytic subgroup of $G$.

Proof. $[H, K]$ is generated by elements of the form $h k h^{-1} k^{-1}$, $h \in H$ and $k \in K$. Since $H$ is an analytic subgroup, we can find an arc $\alpha$ in $H$ with $\alpha(0)=e$ and $\alpha(1)=h$. Then $\beta(t)=\alpha(t) k \alpha(t)^{-1} k^{-1}$ is an arc in $[H, K]$ with $\beta(0)=e$ and $\beta(1)=h k h^{-1} k^{-1}$. Hence $[H, K]$ is arcwise connected. Q.E.D.

Corollary 2 (Corollary to Corollary 1). Let G be a Lie group, and let $H$ be an analytic subgroup of $G$. Then the derived sequence of 
subgroups $H^{(1)}=H, H^{(2)}, \cdots$ defined by $H^{(i+1)}=\left[H^{(i)}, H^{(i)}\right]$, and the descending central sequence $H^{1}, H^{2}, \cdots$ defined by $H^{i+1}=\left[H, H^{i}\right]$ are composed of analytic subgroups.

Corollary 3. Let $G$ be a connected simple Lie group, i.e. the Lie algebra $\$$ of $G$ is simple. Let $N$ be a normal subgroup of $G$. If $G \neq N$, then $N$ is contained in the center of $G$.

Proof. Let $N^{a}$ be the arcwise connected component of $N$ containing $e$. Then $N^{a}$ is a normal analytic subgroup of $G$. Since (5) is simple, we have $N^{a}=\{e\}$. For $a$ in $N,\left\{g a g^{-1}, g \in G\right\}$ is an arcwise connected subset of $N$, and reduces to $\{a\}$.Q.E.D.

University of Pennsylvania 